\title{
Cellular Immune Reactivity to Recombinant OspA and Flagellin from Borrelia burgdorferi in Patients with Lyme Borreliosis \\ Complexity of Humoral and Cellular Immune Responses
}

\begin{abstract}
Andreas Krause, ${ }^{\star}$ Gerd R. Burmester, ${ }^{*}$ Anne Rensing, ${ }^{\star}$ Christoph Schoerner,” Ulrich E. Schaible," Markus M. Simon," Peter Herzer," Michael D. Kramer," and Reinhard Wallich **

*Institute of Clinical Immunology and Rheumatology, Department of Medicine III, University of Erlangen-Nürnberg;

${ }^{\ddagger}$ Institute of Clinical Microbiology, University of Erlangen-Nürnberg, W-8520 Erlangen, Germany; ${ }^{\S}$ Max-Planck-Institute for Immunobiology, W-7800 Freiburg, Germany; "Medizinische Poliklinik, W-8000 Munich, Germany; 'Institute for Immunology, W-6900 Heidelberg, Germany; and ${ }^{* *}$ the German Cancer Research Center, W-6900 Heidelberg, Germany
\end{abstract}

\begin{abstract}
Patients with Lyme borreliosis (LB) usually develop a vigorous $T$ cell response against the causative pathogen Borrelia burgdorferi, but little is known about the antigens recognized in the cellular response. Therefore, $T$ cell reactivities against whole bacteria, recombinant $31-\mathrm{kD}$ (outer surface protein $\mathbf{A}$, [OspA]), and 41-kD proteins (flagellin) from $B$. burgdorferi were studied in patients with $L B$, non-LB patients, and healthy donors. In parallel, specific antibodies were determined by Western blot analysis. Virtually all patients with LB exhibited marked cellular responses to whole $B$. burgdorferi, which were significantly elevated compared with the control groups in both early and late disease stages. However, analyses using the purified antigens OspA and flagellin revealed considerable heterogeneity in the cellular reactivities among individuals as well as variations during the course of infection. $T$ cell responses to OspA were significantly increased in patients with early LB compared with both control groups whereas in late-stage disease responses only exceeded those of non-LB patients and were not different from normal donors. Cellular immune reactivities to flagellin were significantly higher only in early LB compared with both control groups. Reciprocally, several control subjects demonstrated marked cellular responses to OspA and flagellin, suggesting that reactions to these proteins may not always be related to $L B$. T cell reactivity did not correlate well with the presence of specific antibodies. Almost all seropositive patients in both early and late stage $\mathrm{LB}$ had serum antibodies against flagellin, but antibodies to OspA were detectable only in a subset of late LB sera. These data demonstrate the complexity of the humoral and the cellular immune responses to components of $B$. burgdorferi. ( $J$. Clin. Invest. 1992. 90:1077-1084.) Key words: Lyme disease $\bullet T$ cell response • outer surface protein A • diagnosis - Western blot
\end{abstract}

Address correspondence to Gerd R. Burmester, M.D., Institute of Clinical Immunology and Rheumatology, Department of Medicine III, Krankenhausstrasse 12, W-8520 Erlangen, Germany.

Received for publication 19 March 1991 and in revised form 16 March 1992.

J. Clin. Invest.

(c) The American Society for Clinical Investigation, Inc. $0021-9738 / 92 / 09 / 1077 / 08 \quad \$ 2.00$

Volume 90, September 1992, 1077-1084

\section{Introduction}

Lyme borreliosis (LB) ${ }^{1}$ is a multisystemic inflammatory disorder caused by the spirochete Borrelia burgdorferi (1-3). Previous studies have shown that most patients with LB develop an early and strong cellular immune reactivity to $B$. burgdorferi, which in early stages often precedes an only slowly evolving humoral response (4-7). Although the spectrum of specific antibodies against $B$. burgdorferi has been recognized in several studies (8-12), only little is known about the immunodominant $T$ cell antigens. Two major proteins of $B$. burgdorferi, the outer surface protein A (OspA) and flagellin, appear to be of particular interest. The species-specific OspA is a $31-\mathrm{kD}$ protein that is expressed in large amounts on the outer membrane of $B$. burgdorferi. OspA has been described to be antigenically variable between different isolates of $B$. burgdorferi $(11,13$, 14). In mice experimentally inoculated with whole borreliae or with recombinant protein, OspA induces a vigorous humoral immune response $(15,16)$. In the severe combined immunodeficiency mouse model for LB (17), the manifestation of the disease could be prevented or mitigated by the application of anti-OspA antibodies when administered together with the causing agent $(16,18)$. Moreover, active immunization of immunocompetent mice with recombinant OspA induced the production of anti-OspA antibodies and protected from challenge with $B$. burgdorferi, making OspA a possible candidate for a vaccine $(15,16)$. However, in humans the humoral response to OspA as detected by immunoblotting is quite rare, and if present develops only at later stages $(3,5,8,9,12)$. In contrast, antibodies to flagellin, a 41-kD protein associated with the flagella of $B$. burgdorferi, are detectable in nearly all patients with LB even in early stages of the disease $(19,20)$. However, flagellin from $B$. burgdorferi shows high protein sequence homologies with similar proteins from other spirochetes and bacteria not related to $B$. burgdorferi, which may lead to serological cross-reactivities (19, 21-24).

Recently, the genes for OspA (from the German isolate ZS7) (25) and for flagellin (from the American isolate B31) (22) have been cloned by ourselves and by other groups (23, 26) and proteins have been produced in a recombinant, highly purified form $(16,22)$. Based upon our previous findings of high $\mathrm{T}$ cell responses towards whole $B$. burgdorferi bacteria

1. Abbreviations used in this paper: dpm, disintegration per minute; HD, healthy donors; IFA, immunofluorescence assay; LB, Lyme borreliosis; OspA, outer surface protein A. 
(4), the objective of this investigation was to further define the differential cellular immune reactivities towards OspA and flagellin in patients with different clinical stages of LB. These findings were compared with the humoral responses as determined by immunofluorescence tests and Western blot analysis using the two domestic $B$. burgdorferi strains ZS7 and PKo 2-85.

The results obtained demonstrate that patients with early and late infections of LB show a significantly elevated cellular immune response to $B$. burgdorferi, which is in part directed against OspA and flagellin. Furthermore, the data provide evidence for a dissociation between the presence of $B$. burgdorfer $i$ specific antibodies and $T$ cell reactivities in early and late stages of LB.

\section{Methods}

Patients and control subjects. In 36 patients with LB and 25 control subjects the cellular immune response to $B$. burgdorferi antigens was studied. The clinical characteristics of patients and controls are summarized in Table I. All individuals tested were newly entered into the study and therefore represent populations additional to the ones previously reported (4).

11 patients had early infections (disease stages 1 and 2 according to Steere [3]) at the time of study (group 1$)$ with erythema migrans ( $n$ $=7$ ), lymphadenosis cutis benigna $(n=2)$, meningopolyneuritis ( $n$ $=1$ ), and chorioditis $(n=1)$. Of interest, the latter patient later developed severe arthritis and $B$. burgdorferi was cultured from synovectomy material. Six of the 11 patients had detectable antibodies against B. burgdorferi in an immunofluorescence assay (IFA) (4). The time elapsed between a recognized tick or insect bite and the examination of these patients ranged from 3 to $90 \mathrm{~d}$.

The remaining $25 \mathrm{LB}$ patients suffered from late stage disease (group 2) (3), including Lyme arthritis $(n=23)$ and localized scleroderma like skin lesions $(n=2)$. 20 patients were seropositive and 5 were seronegative in the IFA. In the seronegative patients the diagnosis of late LB was based on a typical history of an erythema migrans in four patients, among these was one patient with a complete heart block. One additional seronegative patient had been seropositive before therapy with antibiotics but had converted to seronegative after therapy despite persistance of clinical symptoms. Of the seropositive patients seven had additional previous or concomitant symptoms of LB: two had a history of a recognized erythema migrans, one had acrodermatitis, and four had had typical meningopolyneuritis with specific antibodies in the spinal fluid. In the sera of the remaining patients high IgG antibody titers ( $\geq 1: 256$ in IFA) against $B$. burgdorferi were detectable and in three individuals specific IgM antibodies were found. $B$. burgdorferi was cultured from a synovectomy specimen from one patient, and five individuals showed improvement after ceftriaxone therapy.
Most LB patients of both groups complained about additional constitutional symptoms of different intensities. 2 patients of group 1 and 13 patients of group 2 , including the 5 seronegative individuals, had previously been treated with antibiotics, generally doxycycline or penicillin. However, in all patients symptoms of active LB were still present at the time of investigation, indicating active disease.

Two control groups were established. One group (group 3) consisted of 13 patients with different inflammatory disorders who had initially attended our clinic because of suspected LB but in whom LB was subsequently excluded. Of these, three had RA according to the American College of Rheumatology criteria (27), seven presented with HLA B27-associated reactive arthritis, two had inflammatory skin lesions (one lichen sclerosus et atrophicus, one allergic skin reaction ) not related to borrelia infections, and one suffered from a neuritis nervi optici. In five patients (two seropositive RA, two classical Reiter's syndrome associated with chlamydial infections, one neuritis nervi optici), significant amounts of IgG antibodies against $B$. burgdorferi were determined, but there were no anamnestic or clinical criteria for active LB. Moreover, none of the patients had responded to recommended regimens of antibiotic treatment.

The second control group (group 4) included 12 healthy individuals. All healthy donors (HD) were negative for serum antibodies against $B$. burgdorferi. Members of our laboratories who work with $B$. burgdorferi were excluded from this group (28).

Expression and purification of recombinant $B$. burgdorferi proteins. Escherichia coli transformed by the bacterial expression vector pUEX1 and subsequently expressing OspA or 41-kD flagellin were grown overnight at $30^{\circ} \mathrm{C}$ in $10 \mathrm{ml}$ Luria-Bertani broth with ampicillin (29). $1 \mathrm{ml}$ of overnight cultures was expanded in $100 \mathrm{ml}$ of medium and grown at $30^{\circ} \mathrm{C}$ with good aeration to a density of $8 \times 10^{7}$ cells $/ \mathrm{ml}\left(\mathrm{A}_{600}=0.2\right)$. Expression of recombinant $41-\mathrm{kD}$ flagellin was achieved by transferring the cells to $42^{\circ} \mathrm{C}$ for $2 \mathrm{~h}$. After washing the cells in STE buffer ( 10 $\mathrm{mM}$ Tris, $100 \mathrm{mM} \mathrm{NaCl}, 1 \mathrm{mM}$ EDTA, $\mathrm{pH} \mathrm{8.0)}$ ) the pellet was resuspended in $0.6 \mathrm{ml}$ lysis buffer ( $25 \%$ sucrose $/ 50 \mathrm{mM}$ Tris pH 8.0 ). $150 \mu \mathrm{l}$ of lysozyme $(10 \mathrm{mg} / \mathrm{ml})$ were added and the mixture was incubated for $15 \mathrm{~min}$ on ice followed by another $15 \mathrm{~min}$ incubation on ice with $18 \mu \mathrm{l}$ DNaseI $(10 \mathrm{mg} / \mathrm{ml})$ and $5 \mu \mathrm{l} 1 \mathrm{M} \mathrm{MgCl}$. Finally, $250 \mu \mathrm{l}$ of $4 \times$ detergent mix ( $1 \%$ Triton X-100, 0.5\% Deoxycholate, $0.1 \mathrm{M} \mathrm{NaCl}, 10 \mathrm{mM}$ Tris, $\mathrm{pH} 7.4$ ) were added and incubated for $5 \mathrm{~min}$ on ice. After centrifugation, pellets were washed twice in buffer A $(50 \mathrm{mM}$ Tris, $50 \mathrm{mM}$ $\mathrm{NaCl}, 1 \mathrm{mM}$ EDTA, pH 8.0), resuspended with 9 vol of buffer A containing $8 \mathrm{M}$ urea and incubated for $1 \mathrm{~h}$ at room temperature. The samples were diluted with nine parts of buffer B ( $50 \mathrm{mM} \mathrm{KH}_{2} \mathrm{PO}_{4^{-}}$$\mathrm{K}_{2} \mathrm{HPO}_{4}, \mathrm{pH} 10.7,50 \mathrm{mM} \mathrm{NaCl}, 1 \mathrm{mM}$ Tris) and stirred for $30 \mathrm{~min}$ at room temperature while keeping the $\mathrm{pH}$ at 10.7 by adding $\mathrm{KOH}$. After adjusting the $\mathrm{pH}$ of the solution to 7.0 by the addition of $\mathrm{HCl}$, the samples were dialyzed overnight in the cold against buffer $A$. Solutions were centrifuged for $10 \mathrm{~min}$ at $10,000 \mathrm{~g}\left(4^{\circ} \mathrm{C}\right)$, and the supernatants were further purified by antibody affinity chromatography. Recombinant OspA produced by $E$. coli cells was released into the culture supernatant and enriched by antibody affinity chromatography. Solutions

Table I. Clinical Characteristics of Patients and Control Subjects

\begin{tabular}{|c|c|c|c|c|c|}
\hline Group & $n$ & $\begin{array}{l}\operatorname{Sex} \\
(\mathrm{m} / \mathrm{f})\end{array}$ & $\begin{array}{l}\text { Mean age } \\
\text { (range) }\end{array}$ & $\begin{array}{l}\text { Immunofluorescence } \\
\text { assay \% positive }\end{array}$ & $\begin{array}{l}\text { Previous } \\
\text { antibiotic therapy }\end{array}$ \\
\hline & \multicolumn{4}{|c|}{$y r$} & \\
\hline Patients with early Lyme borreliosis* & 11 & $2 / 9$ & $40(25-75)$ & 55 & 2 \\
\hline Patients with late Lyme borreliosis ${ }^{\ddagger}$ & 25 & $17 / 8$ & $49(15-78)$ & 80 & 13 \\
\hline Non-Lyme borreliosis patients ${ }^{\S}$ & 13 & $7 / 6$ & $40(12-69)$ & 38 & 5 \\
\hline Healthy donors & 12 & $8 / 4$ & $33(23-76)$ & 0 & - \\
\hline
\end{tabular}

* Erythema migrans: $n=7$; lymphadenosis cutis benigna: $n=2$; meningopolyneuritis: $n=1$; chorioiditis: $n=1$. ${ }^{\ddagger}$ Arthritis: $n=23$; localized scleroderma-like skin lesions: $n=2$. ${ }^{\S}$ Rheumatoid arthritis: $n=3$; reactive arthritis: $n=7$; inflammatory skin lesions: $n=2$; neuritis nervi optici: $n=1$. 
containing recombinant $B$. burgdorferi proteins were centrifuged at $100,000 \mathrm{~g}$ for $1 \mathrm{~h}$ and passed over two columns in series at a flow rate of $20 \mathrm{ml} / \mathrm{h}$ : first, a mouse IgG-Sepharose column $(2 \mathrm{ml}$ at $5 \mathrm{mg} / \mathrm{ml})$ to absorb some contaminants and to filter out any particulate material, and second, a LA-2 (for OspA) (23) or a LA-21 (for flagellin) (22) monoclonal antibody column $(3 \mathrm{ml}$ at $10 \mathrm{mg} / \mathrm{ml})$. The column was washed with five column volumes of PBS and the bound material was then eluted using $0.1 \mathrm{M}$ glycine $/ \mathrm{HCl}, \mathrm{pH}$ 3.0. Finally, the recombinant antigens purified by chromatography were dialyzed against PBS (16). Purity of antigens was tested by SDS-PAGE and silver staining, revealing only very faint contaminating bands. Moreover, cellular immune responses were studied initially using whole urea lysates of transformed $E$. coli and demonstrated only minor $\mathrm{T}$ cell reactivities that were not different between normal donors and patients.

Lymphocyte proliferation assay. The lymphocyte proliferation assay was performed as described previously (4). PBMCs were isolated from heparinized peripheral venous blood by standard centrifugation on a Ficoll-Hypaque (Nycomed Pharma, Oslo, Norway) gradient. They were resuspended in RPMI 1640 medium (Biochrom KG, Berlin, Germany) supplemented with L-Glutamin ( $2 \mathrm{mM}$, Sigma Chemical Co., St. Louis, MO), Hepes buffer ( $15 \mathrm{mM}$, Gibco Laboratories, Eggenstein, Germany), penicillin/streptomycin (100 IU / ml and 100 $\mu \mathrm{g} / \mathrm{ml}$, respectively; Gibco Laboratories), and $10 \%$ autologous serum and cultured in triplicate at a concentration of $1 \times 10^{5}$ cells/well in round-bottom microtiter plates (Nunc, Roskilde, Denmark). Cells were stimulated with whole $B$. burgdorferi (German isolate PKo 2-85, kindly provided by Dr. Vera Preac-Mursic, Max-von-Pettenkofer-Institute, Munich, Germany) (30) at concentrations of $1 \times 10^{5}$ and 1 $\times 10^{6}$ bacteria/well (corresponding to 0.3 and $3 \mu \mathrm{g}$ protein/well, respectively) or with recombinant $31-$ and $41-\mathrm{kD}$ proteins at final concentrations of $10,1,0.1$, and $0.01 \mu \mathrm{g} / \mathrm{ml}$. In previous experiments the $T$ cell nature of the cellular response to $B$. burgdorferi antigens had already been demonstrated since only purified $T$ cells but not the non$\mathrm{T}$ cell fraction proliferated in response to stimulation with $B$. burgdorferi (4). Control cultures received medium alone or ultrasonicated Treponema phagedenis (Behringwerke, Marburg, Germany) at 7 and $0.7 \mu \mathrm{g} /$ well. The latter spirochete is of the same family as $B$. burgdorferi and was used as a control antigen. All antigen concentrations had been tested in dose-response experiments and had been found to induce optimal and maximum stimulations in either of the concentrations mentioned above. Therefore, various concentrations were used throughout the experiments. Cultures were incubated at $37^{\circ} \mathrm{C}$ in a humidified atmosphere with $5 \% \mathrm{CO}_{2}$ for $6 \mathrm{~d}$. Subsequently, $1 \mu \mathrm{Ci}$ of $\left[{ }^{3} \mathrm{H}\right]$ thymidine (sp act $2 \mathrm{Ci} / \mathrm{mmol}$; Amersham Buchler, Braunschweig, Germany) was added to each well for $18 \mathrm{~h}$. Cells were harvested semiautomatically and tritiated thymidine incorporation was determined in a liquid scintillation counter (LKB Wallac, Turku, Finland). Results were expressed as average disintegration per minute ([dpm] quench correction by an internal standard) of triplicate wells. $\Delta \mathrm{dpm}$ was calculated by subtracting the control values with medium alone from the dpm obtained in the presence of antigen. In all experiments, for each of the stimuli, i.e., whole borreliae, sonicated $T$. phagedenis, and recombinant proteins, only the maximum values of all concentrations tested will be shown.

Western blot analysis. Sera from all 36 patients with LB (groups 1 and 2) and from 12 non-LB patients (group 3) were obtained on the same day as the PBMCs and were subjected to immunoblot analysis for specific antibodies against $B$. burgdorferi. Since there may be antigenic heterogeneity between borrelia strains, resulting in different results in the immunoblots $(11,12,31)$, two different german $B$. burgdorferi isolates PKo (30) and ZS7 (17) (from which the gene for the recombinant OspA had been cloned) were used as antigens. In brief, whole borreliae were lysed in Tris/glycerin/SDS buffer, the protein concentration was determined, and $5 \mu \mathrm{g}$ protein/lane were separated by SDSPAGE with 10 or $12 \%$ polyacrylamide running gels as described by Laemmli (32). Proteins were transferred to nitrocellulose and, after blocking with $0.1 \%$ bovine serum albumin or with $3 \%$ gelatin, incubated with the sera at a 1:100 dilution. Bound immunoglobulins were visualized with appropriate peroxidase-conjugated goat anti-human immunoglobulin antibodies. Based upon own observations with patient and control sera (unpublished data) and according to the suggestions of Zöller et al. (12) as well as Grodzicki and Steere (9), blots fulfilling one of the following criteria were considered positive: $(a)$ in early disease stages a clear $41-\mathrm{kD}$ band and at least two additional bands with one band corresponding to the $21-, 30-, 60-$, or $87-\mathrm{kD}$ protein; $(b)$ in late LB five or more antibodies against $B$. burgdorferi proteins with a strong 18-, 21-, 23-, 30-, 41-, 75-, or 87-kD band. For further evaluation, special attention was placed on the presence of antibodies against OspA and flagellin.

Immunofluorescence assay. The IFA was performed as described previously (4). All sera were preabsorbed with $T$. phagedenis before testing in order to eliminate cross-reacting antibodies. After this procedure, the IFA was highly specific for $B$. burgdorferi, and there virtually was no cross-reaction to other spirochetes, especially Treponema pallidum. In a random Bavarian (Franconian) population $(n=334)$ matched for similar living areas as the patient population, $276(83 \%)$ were seronegative, $33(10 \%)$ had titers of $1: 16,6(2 \%)$ of $1: 32,10(3 \%)$ of $1: 64$, and $9(3 \%)$ of $\geq 1: 128$. Therefore, in the clinical situation, titers from 1:64 on were regarded as indicative of a definite serological reaction (10). For the detection of specific IgM antibodies, sera were additionally pretreated with rheumatoid factor absorbent (Behringwerke) to prevent false-positive results due to rheumatoid factor absorbent or false-negative results because of competitive inhibition by specific IgG.

Statistical analysis. Data are presented as median values (range) unless otherwise indicated. The proliferation values of the four groups tested were compared by the Mann-Whitney $U$ test, and $P$ values of $<0.05$ were considered to be significant. Correlations between response values to different antigens within the study groups were determined by the Spearman rank correlation test. Correlation coefficients were considered significant at $P<0.01$.

\section{Results}

$T$ cell response towards whole $B$. burgdorferi and $T$. phagedenis. The cellular immune responses to in vitro stimulation with whole $B$. burgdorferi bacteria were significantly elevated in patients with early (median $37,700 \mathrm{dpm}$ ) and with late LB (median 40,000 dpm) as compared with non-LB patients (median 13,200 dpm, $P<0.0005$ ) or HD (median 18,000 dpm, $P$ $<0.002$ ) (Fig. $1 A$; Table II). This increased lymphoproliferative response did not correlate with the presence of specific antibodies against $B$. burgdorferi and was already detectable as early as $2 \mathrm{~d}$ after the onset of the erythema migrans. No significant differences in proliferation values were observed either between patients with early and late infections or between nonLB patients and HD. Despite highly significant differences in response rates between LB patients and control subjects, the majority of control individuals showed responses to whole $B$. burgdorferi, which, however, generally were marginal in magnitude (Fig. $1 A$ ).

In contrast to $B$. burgdorferi, stimulation of PBMC with $T$. phagedenis generally resulted in moderate proliferative responses in all four groups tested. Only in patients with LB and irrespective of the disease stage did $\mathrm{T}$ cell proliferation induced by $B$. burgdorferi always exceed responses elicited by stimulation with $T$. phagedenis (early: median values 37,700 vs. 7,800 dpm, $P<0.004$, late: 40,000 vs. $9,400 \mathrm{dpm}, P<0.0001$ ) (Fig. $1, A$ and $B$; Table II).

$T$ cell responses to recombinant $O$ sp $A$ and flagellin. Fig. $1 C$ demonstrates that the cellular immune responses to OspA were significantly increased in patients with early LB (median 


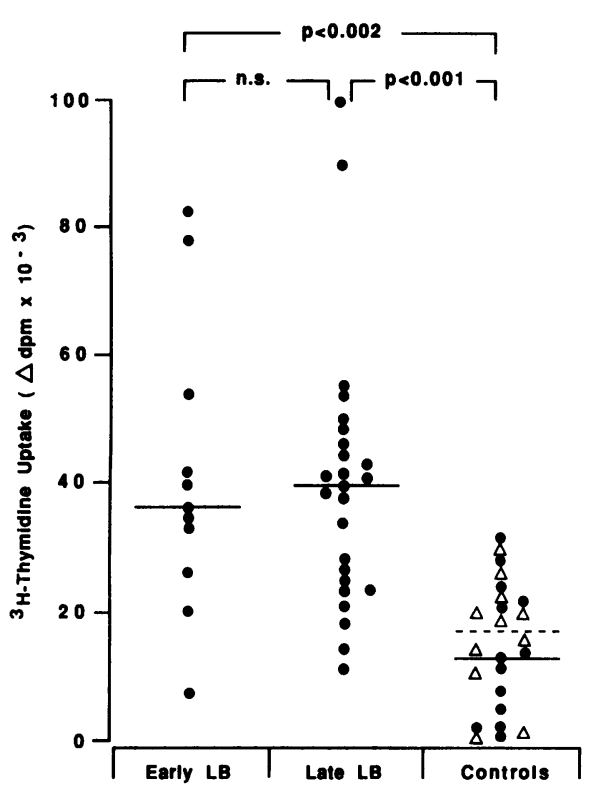

c

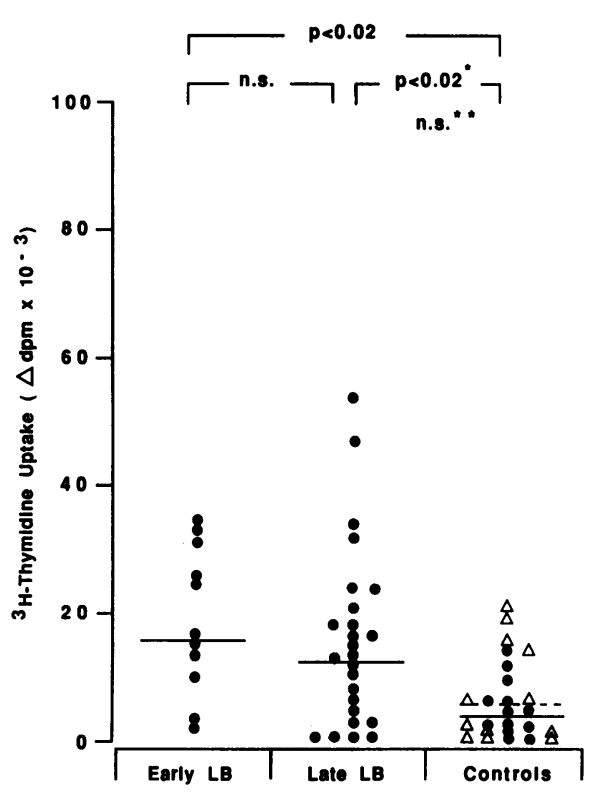

B

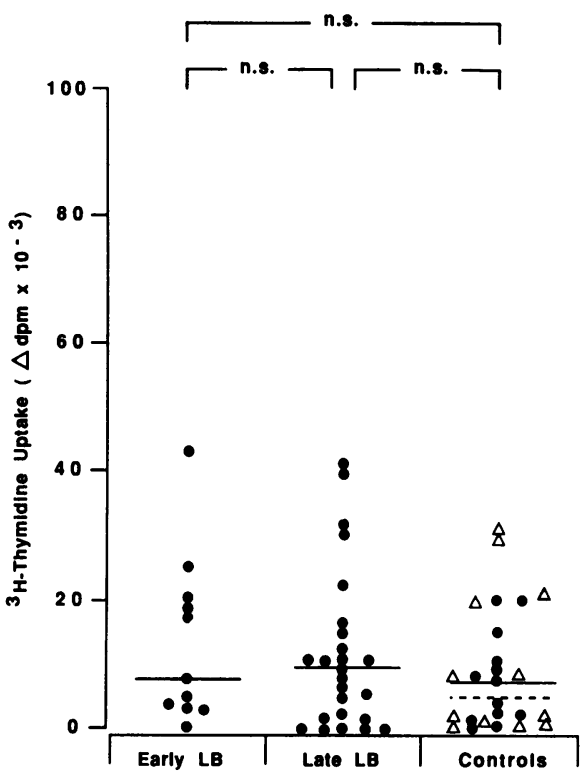

D

41 KD Protein (Flagellin)

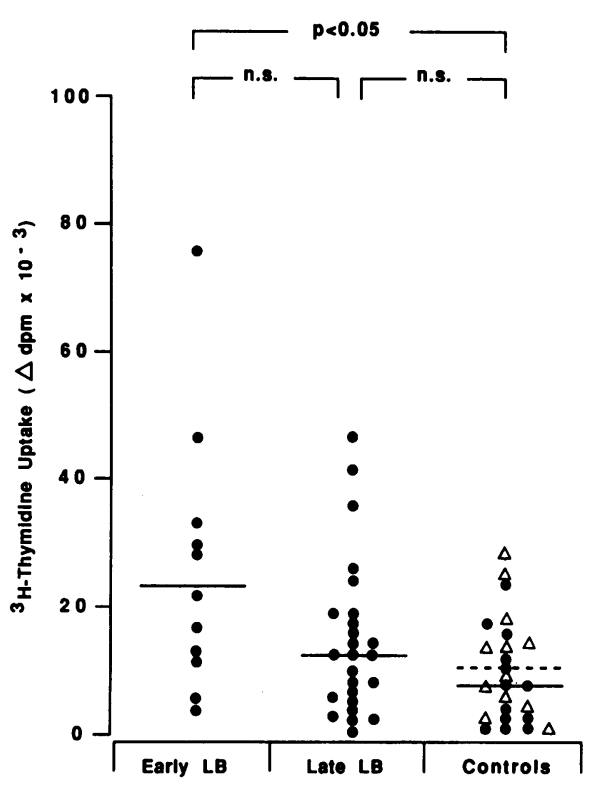

Figure 1. Proliferative responses of PBMC, to $(A)$ whole $B$. burgdorferi, (B) $T$. phagedenis, $(C)$ recombinant 31-kD protein (OspA), and (D) recombinant $41-\mathrm{kD}$ protein (flagellin). PBMC from patients with early LB $(n=11)$, late LB $(n=25)$, patients with various inflammatory disorders not related to LB (non-LB patients) (Controls $\bullet ; n=13$ ), and from HD (Controls $\Delta ; n=12$ ) were stimulated with different concentrations of the antigens in 7-d cultures. Proliferation was determined by tritiated thymidine incorporation, maximum proliferation values are given in $\Delta \mathrm{dpm}$ (dpm of antigen stimulated cultures minus dpm of control cultures with medium alone). The horizontal bars show the median proliferative responses in each group (hatched bars, HD). Response values of the four groups tested were compared by the MannWhitney $U$ test (*late LB vs. non-LB patients, **late LB vs. HD).
$16,000 \mathrm{dpm}$ ) compared with non-LB patients (median 4,400 dpm, $P<0.002$ ) and HD (median 5,000 dpm, $P<0.02$ ) (Fig. $1 C$; Table II). There were only two patients with very low response values of 1,900 and $3,000 \mathrm{dpm}$, whereas the majority of patients showed marked responses of $>10,000 \mathrm{dpm}$. In patients with late infection, the proliferation values (median $13,500 \mathrm{dpm}$ ) were significantly higher compared with non-LB patients $(P<0.02)$ but did not significantly exceed the responses obtained in HD (group 4) $(P=0.11)$. Seven patients of group 2 exhibited only weak responses of $<5,000 \mathrm{dpm}$, and 16 individuals responded well with $>10,000 \mathrm{dpm}$. Of special interest, one patient in the non-LB group and four HD (all five of them seronegative) showed marked $T$ cell reactivities to OspA exceeding the median value of late LB patients.
In contrast, $\mathrm{T}$ cell responses to flagellin (Fig. $1 \mathrm{D}$ ) were significantly elevated only in patients with early LB (median $22,700 \mathrm{dpm}$ ) and exceeded the proliferation values of the two control groups (group 3: median 8,000 dpm, $P<0.008$; group 4: median $11,300 \mathrm{dpm}, P<0.05$ ). Responses of late LB patients (median 12,300) did not significantly differ from those of patients with early LB or of the control groups (Table II). Only two patients with early LB, eight individuals with late infection, six non-LB patients, and three HD showed low responses of $\leq 5,000 \mathrm{dpm}$, whereas the majority of individuals tested responded well ( $>10,000 \mathrm{dpm}$ ) to flagellin. One individual with early and six individuals with late infections did not significantly respond to either OspA or flagellin; however, five of these seven patients elicited marked proliferative reactions 
Table II. Statistical Analysis of Differences

of Proliferation Values of PBMC* between the Four Groups Studied (Mann-Whitney U Test)

\begin{tabular}{lcccc}
\hline & & \multicolumn{2}{c}{ Responses to } & \\
\cline { 3 - 4 } Comparison of groups & B. burgdorferi & T. phagedenis & OspA & Flagellin \\
\hline Early LB vs. late LB & $\mathrm{NS}^{\S}$ & $\mathrm{NS}$ & $\mathrm{NS}$ & $\mathrm{NS}$ \\
Early LB vs. non-LB & $0.0005^{\|}$ & $\mathrm{NS}$ & 0.002 & 0.008 \\
Early LB vs. HD & 0.002 & $\mathrm{NS}$ & 0.02 & 0.05 \\
Late LB vs. non-LB & 0.0001 & $\mathrm{NS}$ & 0.02 & NS \\
Late LB vs. HD & 0.0001 & $\mathrm{NS}$ & $\mathrm{NS}$ & $\mathrm{NS}$ \\
Non-LB vs. HD & $\mathrm{NS}$ & $\mathrm{NS}$ & $\mathrm{NS}$ & $\mathrm{NS}$ \\
& & & & \\
\hline
\end{tabular}

* Proliferative responses of PBMC were determined by a lymphocyte proliferation assay (for methods see Fig. 1). ${ }^{\ddagger}$ Early LB (group 1): patients with early Lyme borreliosis, late LB (group 2): patients with late Lyme borreliosis, non-LB (group 3): patients with inflammatory disorders not related to Lyme borreliosis, HD (group 4): healthy donors. For further definition of groups 1 to 4 , see Table I. ${ }^{8} \mathrm{NS}$, not significant $(P>0.05)$. " $P$ values.

to stimulation with whole $B$. burgdorferi. In the control groups, $T$ cells derived from 4 of 13 non-LB patients and 6 of $12 \mathrm{HD}$ showed response values to flagellin above the median value of late LB patients (Fig. $1 \mathrm{D}$; Table II).

Table III shows that in patients with LB there were strong, consistent, and significant correlations between $\mathrm{T}$ cell responses to the spirochetal antigens tested. In contrast, in healthy donors and non-LB patients generally no consistent correlations were observed. Here, only responses to $B$. burgdorferi and $T$. phagedenis correlated significantly ( $r=0.70, P$ $<0.01$ ) in non-LB patients, whereas in HD a very strong and highly significant correlation was observed between proliferation values after stimulation with $T$. phagedenis and OspA ( $r$ $=0.91, P<0.001)$.

Humoral immune responses to $B$. burgdorferi proteins. Of the six patients with early LB who were seropositive in the IFA, three showed a restricted antibody pattern with bands corresponding to the $41-\mathrm{kD}$ protein and one or two additional bands, whereas the remaining three individuals showed an extended pattern of more than five antigens recognized (Fig. 2). Two of five individuals with negative IFA immunoblots were considered positive according to the criteria mentioned in Methods. These sera had also been analyzed without preabsorption, again yielding negative results in the IFA. Antibodies against the $31-\mathrm{kD}$ protein were not detectable and there were no major differences between blots using strains PKo or ZS7.

In the group of patients with late LB (group 2), all of the 20 IFA-positive patients also had positive immunoblots revealing at least 5 , and in some cases $>10$, intense bands. All but 3 seropositive patients exhibited antiflagellin antibodies, and 16 individuals showed antibodies against a $87-\mathrm{kD}$ protein. Antibodies against OspA of PKo were found in only four patients; antibodies against OspA of ZS7 were found in only three individuals. In these patients, $T$ cell proliferation values to OspA were $24,300,14,600,12,400 \mathrm{dpm}$, and, interestingly, in one patient $\mathrm{T}$ cells did not react to OspA at all. It is noteworthy that in most individuals antibodies against a $30-\mathrm{kD}$ protein were observed as has been described by others (12). This protein could be clearly distinguished from OspA with sera-containing antibodies against both proteins and by additional experiments using monoclonal antibodies. Moreover, on most blots OspA could easily be recognized by a light, "negatively stained" band with missing background staining activity of the nitrocellulose strip due to the presence of high amounts of this protein (Fig. 2).

In group 3 (non-LB patients), positive immunoblots were observed in all five seropositive patients. These blots generally showed bands corresponding to flagellin and four or more additional bands. As in early LB, antibodies against OspA were not detectable. In the majority of the seronegative patients with LB, non-LB patients and HD weak 41-kD bands and sometimes faint additional bands were observed (Fig. 2).

Complexity of humoral and cellular responses. In Table IV, results of lymphocyte proliferation assays and serological analyses of individual patients are outlined, demonstrating the complexity of cellular and humoral responses against components of $B$. burgdorferi. In the early LB group (group 1), i. e., patients 2,3 , and 4 exhibited high $T$ cell reactivities but differed remarkably in antibody responses. In contrast, patient 1 showed a weak cellular response in spite of a high antibody titer. In patients with late infections (group 2), almost all combinations of high and low responses could be observed and no consistent correlations between $\mathrm{T}$ cell reactivities to $B$. burgdorferi proteins or between humoral and cellular responses were detectable. Obviously, the cellular immune response to $B$. burgdor-

Table III. Correlation Analysis (Spearmann Rank Correlation) of Proliferative Responses of PBMC to B. burgdorferi, T. phagedenis, and to Recombinant OspA and Flagellin from B. burgdorferi within the Four Groups Tested

\begin{tabular}{|c|c|c|c|c|}
\hline \multirow[b]{2}{*}{ Comparison of responses to } & \multicolumn{3}{|c|}{ Groups } & \multirow[b]{2}{*}{ HD } \\
\hline & Early LB* & Late LB & Non-LB & \\
\hline B. burgdorferi vs. $T$. phagedenis & $0.7497^{\ddagger 8}$ & $\mathbf{0 . 7 2 5 8} 8^{\| \prime}$ & $0.7042^{\S}$ & 0.4561 \\
\hline B. burgdorferi vs. OspA & 0.6155 & $0.4834^{\S}$ & 0.5882 & 0.5028 \\
\hline B. burgdorferi vs. flagellin & $0.7251^{\S}$ & $0.7205^{\|}$ & 0.3571 & 0.4976 \\
\hline T. phagedenis vs. OspA & $0.8375^{\prime \prime}$ & $\mathbf{0 . 8 0 3 3} 3^{\|}$ & 0.6273 & $0.9088^{\| \prime}$ \\
\hline T. phagedenis vs. flagellin & $0.9031^{11}$ & $0.7996 "$ & 0.2172 & 0.3314 \\
\hline OspA vs. flagellin & $0.7121^{8}$ & 0.7296 & 0.0396 & 0.2871 \\
\hline
\end{tabular}

* For definition of groups 1 to 4 see Tables I and II. ${ }^{\ddagger}$ Correlation coefficient.

Significant correlations are written in bold numbers and are indicated by ${ }^{\S}(P<0.01)$ and " $(P<0.001)$. 


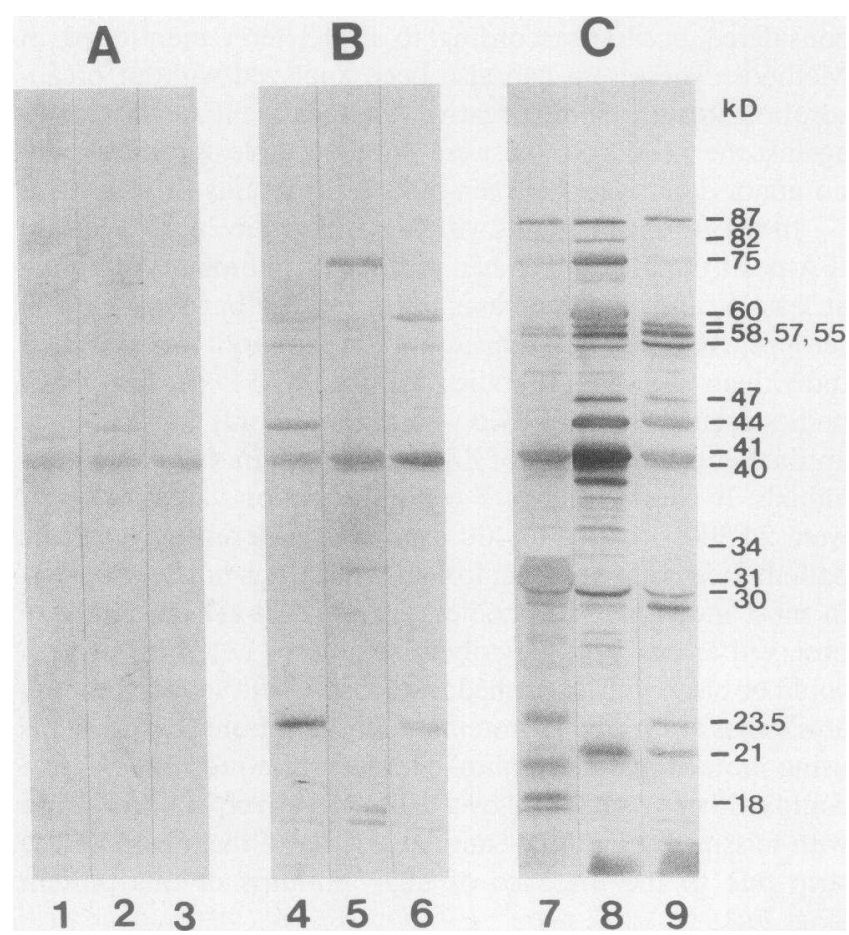

Figure 2. Western blot analysis of antibodies to $B$. burgdorferi proteins in sera from $(A) \mathrm{HD}$ (lanes 1-3), $(B)$ patients with early LB (lanes 4-6), and ( $C$ ) patients with late LB (lanes 7-9). B. burgdorferi bacteria were lysed, and $5 \mu \mathrm{g}$ protein per lane were separated by SDS-PAGE with $10 \%$ running gels. Proteins were transferred to nitrocellulose and probed with the sera at 1:100 dilutions. Bound immunoglobulins were detected by peroxidase-conjugated goat antihuman antibodies. Molecular weight markers are shown on the right.

feri did not depend on the IFA titer or on the presence of specific IgM antibodies. As could be observed in the early LB group, patients with almost similar $\mathrm{T}$ cell reactivities against whole $B$. burgdorferi bacteria (patients 8-13) showed marked differences in both cellular and humoral responses against $B$. burgdorferi antigens. Patients 12 and 14 exhibited the highest cellular reactivities against OspA but had negative IFAs and, in the immunoblot, antibodies against this protein were not found. Conversely, $T$ cells of one of the patients with antiOspA antibodies (patient 10) did not respond to stimulation with OspA. In the sera of patients 11,13 , and 16, antibodies against flagellin were not detectable but all three patients showed marked cellular reactions to the $41-\mathrm{kD}$ antigen. However, some patients with strong anti-41-kD bands in Western blot analysis only showed weak or even no cellular reactivities to flagellin (patients 5, 8, and 9). The three non-LB patients with the highest IFA titers (patients 17-19) only demonstrated weak $\mathrm{T}$ cell responses against $B$. burgdorferi. Interestingly, the patient with RA showed almost no cellular antigen response as has been observed in earlier studies (4). In contrast, the patient with the highest cellular response in this group was seronegative in both IFA and Western blot.

\section{Discussion}

The following principle points emerged from this investigation of the cellular immune responses to two of the recombinant major proteins of $B$. burgdorferi, OspA and flagellin, in patients with LB:

(a) Patients with LB showed a significantly elevated T cell response to whole $B$. burgdorferi bacteria compared with nonLB patients and normal controls. This elevated response was already detectable in early disease stages and did not correlate with the presence of antibodies against $B$. burgdorferi in these patients.

(b) The B. burgdorferi-specific T cell responses observed included those directed against the $31-\mathrm{kD}$ (OspA) and the 41$\mathrm{kD}$ (flagellin) proteins of $B$. burgdorferi. However, there was a considerable variation of proliferation values ranging from a lack of $T$ cell proliferation to very high response values. In early infection, cellular responses to both OspA and flagellin significantly exceeded those of both control groups whereas in late infection only responses to OspA were significantly elevated when compared with the non-LB group. In contrast to the control groups, in patients with LB response values to the spirochetal antigens generally showed high correlations.

(c) Irrespective of the disease stage, in vitro $\mathrm{T}$ cell responses to either whole $B$. burgdorferi or to the recombinant proteins did not correlate with the presence of specific antibodies in the patient sera.

Our data corroborate the results of previous studies by Dattwyler et al. (5) and by our group (4), revealing that most patients with LB develop an early and vigorous cellular immune response to the causative agent $B$. burgdorferi that does not correlate with the presence of specific antibodies. However, all previous tests used whole or sonicated bacteria; therefore, only little was known about the $T$ cell responses against individual $B$. burgdorferi antigens. Recently, two major proteins of $B$. burgdorferi, i.e., OspA and flagellin, had been produced in a recombinant form by our group $(22,24)$ and were used for $T$ cell stimulation assays in this study. The data obtained demonstrate that both antigens are already able to induce strong cellular immune reactions in vitro in early stages of the infection; only 1 of 11 patients with early LB did not markedly respond to either protein. However, in both groups of LB patients, T cell proliferative responses were quite variable and ranged from no or marginal to very high responses. Even in late stage disease a considerable number of patients only exhibited weak responses. Though quantitative differences in cellular reactivities may to some extent be due to different compartmentalization of the immune response, these data may indicate a restricted clonal $\mathrm{T}$ cell expansion in infected individuals with differential reactivities to various $B$. burgdorferi antigens. This selective expansion of responding cells may depend on the HLA profile. In this respect, recent findings by Steere et al. (33) are of special interest. These authors documented a high association of chronic LB with certain HLA-DR2 and -DR4 alleles. To address these questions, HLA typing of our study population and analysis of specific $\mathrm{T}$ cell clones are underway in our laboratory.

A particularly interesting finding in the present and in earlier (5) investigations is the complexity of the humoral and the cellular immune responses. Great care was taken to rule out technical problems as a reason for the inability to detect specific antibodies. Both immunofluorescence and Western blot analyses were performed in all individuals tested. Moreover, immunoblots were carried out in two separate laboratories with two different isolates of $B$. burgdorferi. The presence of immunoreactive bacterial proteins was verified using monoclo- 
Table IV. Complexity of Cellular and Humoral Immune Responses to B. burgdorferi, OspA, and Flagellin

\begin{tabular}{|c|c|c|c|c|c|c|c|c|c|}
\hline \multirow[b]{2}{*}{ No. } & \multirow[b]{2}{*}{ Initials } & \multirow[b]{2}{*}{ Age, sex } & \multirow[b]{2}{*}{ Diagnosis } & \multicolumn{4}{|c|}{$\mathrm{LPA}^{*}$} & \multicolumn{2}{|c|}{ Serology } \\
\hline & & & & B. burgd. ${ }^{\ddagger}$ & OspA & Flagellin & T. phaged. ${ }^{\ddagger}$ & IFA-Titer (IgG, IgM) & Immunoblot \\
\hline
\end{tabular}

A. Patients with early Lyme borreliosis (group 1)

\begin{tabular}{|c|c|c|c|c|c|c|c|c|}
\hline E.H. & $75, \mathrm{f}$ & $\mathrm{LACB}^{\S}$ & 8,500 & 9,500 & 2,000 & 3,500 & $1: 256$, neg. & $87,82,46,41,40,39,29$ \\
\hline W.L. & $47, \mathrm{f}$ & Chorioditis & 56,500 & 34,000 & 23,000 & 26,000 & $1: 128$, neg. & $75,58,41,40,30,14$ \\
\hline A.M. & $28, \mathrm{f}$ & $\mathrm{EM}^{\S}$ & 79,000 & 30,500 & 76,500 & 45,500 & neg., neg. & neg. \\
\hline M.P. & $25, \mathrm{~m}$ & EM & 85,000 & 25,000 & 34,500 & 18,500 & $1: 32,1: 16$ & $41,23,20$ \\
\hline
\end{tabular}

B. Patients with late Lyme borreliosis (group 2)

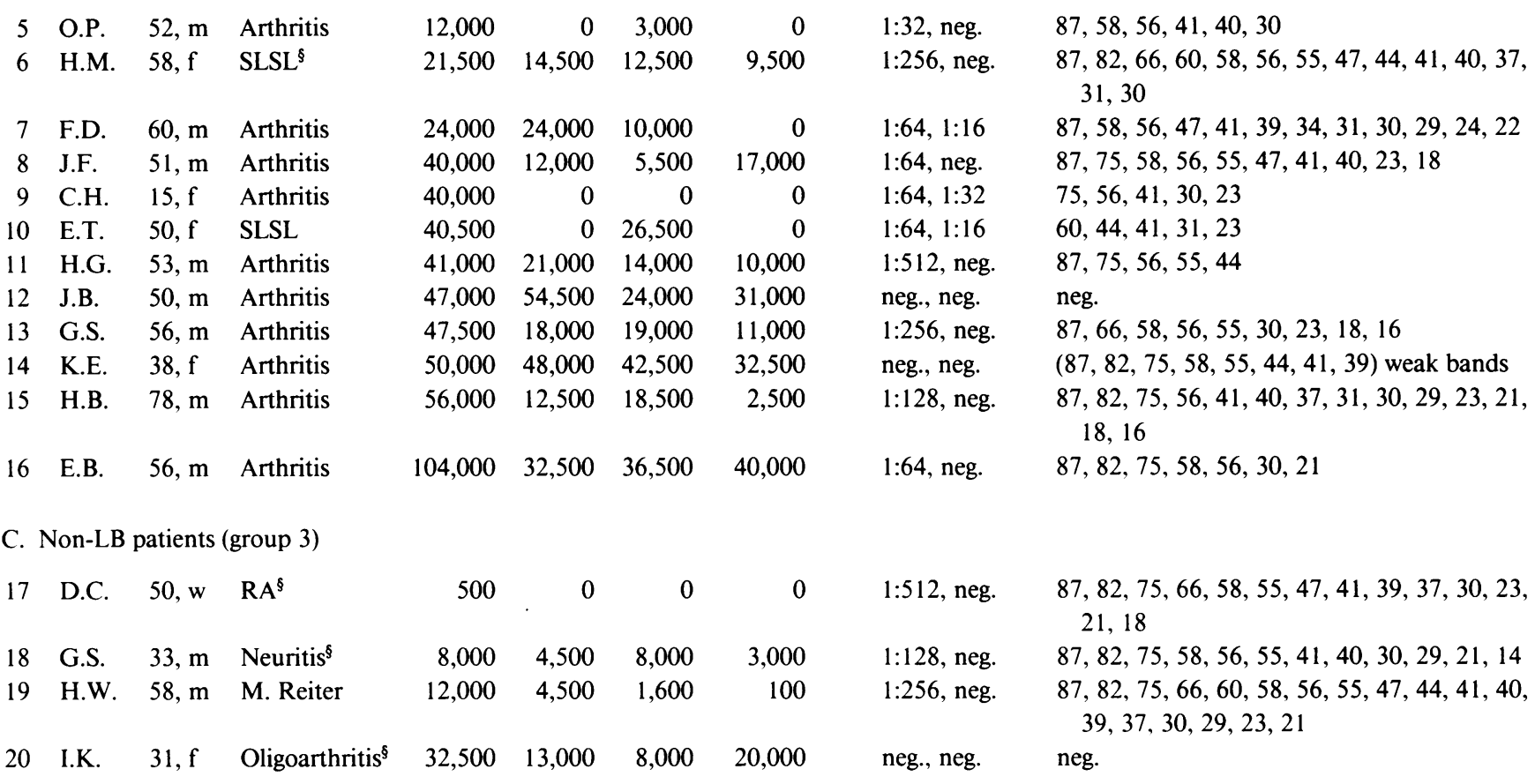

* LPA: lymphocyte proliferation assay, values are given in desintegrations per minute (dpm). ${ }^{\ddagger}$ B. burgd.: Borrelia burgdorferi; $T$. phaged.: Treponema phagedenis. ${ }^{\S}$ LACB: lymphadenosis cutis benigna; EM: erythema migrans; SLSL: scleroderma-like skin lesions; RA: rheumatoid arthritis; neuritis: neuritis n. optici; oligoarthritis: HLA B27-associated seronegative oligoarthritis.

nal antibodies. Sera tested in the IFA were preabsorbed with $T$. phagedenis to increase the specificity of the test $(4,10,11)$. Theoretically, this procedure may have lowered antibody titers in general since cross-reactive epitopes may be the major antigenetic sites. Therefore, Western blot analysis was performed without preabsorption. Comparing both tests, only two sera were identified that were negative in the IFA and positive in the immunoblot. These sera were additionally analyzed in the IFA without preabsorption with $T$. phagedenis, again yielding negative results. With regard to Western blot analysis, there remains the possibility that low concentrations of specific antibodies, especially those directed against OspA, were diluted out by dilution steps necessary to perform immunoblots. However, recently a series of LB sera was tested by our group in a self-developed ELISA using recombinant OspA and flagellin as antigens. In these experiments, similar frequencies of specific antibodies were detected compared with the Western blot in this study (Kramer, M., et al., unpublished data). Furthermore, it is unlikely that strain-specific differences of the OspA protein may account for the inability to detect specific antibodies since the OspA appears to be highly conserved even among isolates from two different continents (15). Thus, the apparent dissociation between $\mathrm{T}$ and $\mathrm{B}$ cell responses, initially described by Dattwyler et al. ( 5 ) and now confirmed by us using recombinant spirochetal antigens, remains to be explored. Possibly, the B. burgdorferi-specific T cells as revealed by in vitro restimulation are in a resting stage in vivo and are not able to provide sufficient signals for B cells to produce antibodies, in particular to OspA. This may in part be due to early antibiotic therapy as described previously $(5,7)$. In fact, in our study all five seronegative patients with late LB had been treated with antibiotics. Therefore, the inability to detect anti-spirochetal antibodies in the serum may be due to a refractory stage of the respective $T$ cells rather than to the absence of previously activated B cells.

The situation of divergence of cellular and humoral responses is even more complicated by the fact that in one of the four individuals with detectable antibodies to OspA there was no $T$ cell response to the same antigen. Clearly, further studies 
are required to gain more insight into the activation of $\mathrm{T}$ cells and B cells in LB.

Analysis of cellular responses in the control groups revealed that $T$ cell reactivity to OspA was not restricted to LB patients but was also detectable in healthy donors and in patients with other inflammatory diseases. In HD there was a highly significant correlation between $T$ cell responses to OspA and to $T$. phagedenis. These results suggest that other saprophytic spirochetes share $\mathrm{T}$ cell epitopes with OspA from $B$. burgdorferi. This assumption is supported by recent observations by Magnarelli et al. (24) and Cooke et al. (34) demonstrating antibody cross-reactivity between $B$. burgdorferi and Treponema, i.e., Treponema denticola. The latter agent is a saprophyte of the human oral mucosa and is frequently found in periodontitis. It may therefore also trigger cellular cross-reactions.

However, despite these possible cross-reactions, it was clearly evident that the T cell reactivity to whole $B$. burgdorfer $i$ and to OspA was significantly elevated in patients with LB. Although OspA and flagellin may not be the ideal antigens to unequivocally link the respective proliferative responses to $B$. burgdorferi, our findings suggest that in the future a panel of standardized recombinant proteins may function as surrogates for whole $B$. burgdorferi. Taken together, the analysis of the $T$ cell reactivity will not only provide significant insights into the pathogenic disease mechanisms but, especially in view of the complexity of the humoral and the cellular immune responses, also appears to be an important tool for the diagnosis of LB.

\section{Acknowledgments}

We thank Dr. R. Winchester, Columbia University, New York, for helpful discussions and thoroughly reviewing the manuscript.

This study was supported in part by the Federal Ministry for Research and Technology (grant $01 \mathrm{KI} 9001$ ). Andreas Krause is a postdoctoral fellow of the Deutsche Forschungsgemeinschaft (grant $\mathrm{Kr}$ 997/1-2).

\section{References}

1. Burgdorfer, W., A. G. Barbour, S. F. Hayes, J. L. Benach, and E. Grunwaldt. 1982. Lyme disease-a tick-borne spirochetosis? Science (Wash. DC). 216:1317-1319.

2. Steere, A. C., R. L. Grodzicki, A. N. Kornblatt, J. E. Craft, A. G. Barbour, W. Burgdorfer, G. P. Schmid, E. Johnson, and S. E. Malawista. 1983. The spirochetal etiology of Lyme disease. N. Engl. J. Med. 308:733-740.

3. Steere, A. C. 1989. Lyme disease. N. Engl. J. Med. 321:586-596.

4. Krause, A., V. Brade, C. Schoerner, W. Solbach, J. R. Kalden, and G. R. Burmester. 1991. T cell proliferation induced by Borrelia burgdorferi in patients with Lyme borreliosis. Autologous serum required for optimal stimulation. $A r$ thritis Rheum. 34:393-402.

5. Dattwyler, R. J., D. J. Volkman, B. J. Luft, J. J. Halperin, J. Thomas, and M. G. Golightly. 1988. Seronegative Lyme disease: dissociation of specific T- and B-lymphocyte responses to Borrelia burgdorferi. N. Engl. J. Med. 319:14411446.

6. Dattwyler, R. J., J. A. Thomas, J. L. Benach, and M. G. Golightly. 1986. Cellular immune response in Lyme disease: the response to mitogens, live Borrelia burgdorferi, NK cell function and lymphocyte subsets. Zentralbl. Bakteriol. Mikrobiol. Hyg. Ser. A. 263:151-159.

7. Dattwyler, R. J., D. J. Volkman, J. J. Halperin, B. J. Luft, J. Thomas, and M. G. Golightly. 1988. Specific immune responses in Lyme disease. Characterization of T cell and B cell responses to Borrelia burgdorferi. Ann. NY Acad. Sci. 539:93-102.

8. Craft, J. E., D. K. Fischer, G. T. Shimamoto, and A. C. Steere. 1986. Antigens of Borrelia burgdorferi recognized during Lyme disease. Appearance of a new immunoglobulin $M$ response and expansion of the immunoglobulin $G$ response late in the illness. J. Clin. Invest. 78:934-939.

9. Grodzicki, R. L., and A. C. Steere. 1988. Comparison of immunoblotting and indirect enzyme-linked immunosorbent assay using different antigen preparations for diagnosing Lyme disease. J. Infect. Dis. 157:790-797.
10. Grellner, W., F. Erbguth, and V. Brade. 1989. Serodiagnostik bei LymeBorreliose: Antikörpertiter und -spezifität im IFT und Western-Blot. Immun. Infekt. 6:189-194.

11. Wilske, B., V. Preac-Mursic, G. Schierz, W. Gueye, P. Herzer, and K. Weber. 1988. Immunochemical analysis of the immune response in late manifestations of Lyme borreliosis. Zentralbl. Bakteriol. Hyg. Ser. A. 267:549-558.

12. Zöller, L., S. Burkard, and H. Schäfer. 1991. Validity of Western immunoblot band patterns in the serodiagnosis of Lyme borreliosis. J. Clin. Microbiol. 29:174-182.

13. Barbour, A. G., R. A. Heiland, and T. R. Howe. 1985. Heterogeneity of major proteins in Lyme disease borreliae: a molecular analysis of North American and European isolates. J. Infect. Dis. 152:478-484.

14. Luft, B. J., W. Jiang, P. Munoz, R. J. Dattwyler, and P. D. Gorevic. 1989. Biochemical and immunological characterization of the surface proteins of Borrelia burgdorferi. Infect. Immun. 57:3637-3645.

15. Fikrig, E., S. W. Barthold, F. S. Kantor, and R. A. Flavell. 1990. Protection of mice against the Lyme disease agent by immunizing with recombinant OspA. Science (Wash. DC). 250:553-556.

16. Simon, M. M., U. E. Schaible, M. D. Kramer, C. Eckerskorn, H. K Müller-Hermelink, and R. Wallich. 1991. Recombinant outer surface protein A from Borrelia burgdorferi induces antibodies protective against spirochetal infection in mice. J. Infect. Dis. 164:123-132.

17. Schaible, U. E., M. D. Kramer, C. Museteanu, G. Zimmer, H. Mossmann, and M. M. Simon. 1990. The severe combined immunodeficiency (scid) mouse. A laboratory model for the analysis of Lyme arthritis and carditis. J. Exp. Med. 170:1427-1432.

18. Schaible, U. E., M. D. Kramer, K. Eichmann, M: Modolell, C. Museteanu, and M. M. Simon. 1990. Monoclonal antibodies specific for the outer surface protein A (OspA) of Borrelia burgdorferi prevent Lyme borreliosis in severe combined immunodeficiency (scid) mice. Proc. Natl. Acad. Sci. USA. 87:3768-3772.

19. Barbour, A. G., and S. F. Hayes. 1986. Biology of Borrelia species. Microbiol. Rev. 50:381-400.

20. Hansen, K., P. Hindersson, and N. S. Pedersen. 1988. Measurement of antibodies to the Borrelia burgdorferi flagellum improves serodiagnosis in Lyme disease. J. Clin. Microbiol. 26:338-346.

21. Barbour, A. G., S. F. Hayes, R. A. Heiland, M. E. Schrumpf, and S. L. Tessier. 1986. A borrelia-specific antibody binds to a flagellar epitope. Infect. Immun. 52:549-554.

22. Wallich, R., S. E. Moter, M. M. Simon, K. Ebnet, A. Heiberger, and M. D. Kramer. 1990. The Borrelia burgdorferi flagellum associated 41-kilodalton antigen (flagellin): molecular cloning, expression and amplification of the gene. Infect. Immun. 58:1711-1719.

23. Gassmann, G. S., M. Kramer, U. B. Gobel, and R. Wallich. 1989. Nucleotide sequence of a gene encoding the Borrelia burgdorferi flagellin. Nucleic Acids Res. 17:3590.

24. Magnarelli, L. A., J. N. Miller, J. F. Anderson, G. R. Riviere. 1990 Cross-reactivity of nonspecific treponemal antibody in serologic tests for Lyme disease. J. Clin. Microbiol. 28:1276-1279.

25. Wallich, R., U. E. Schaible, M. M. Simon, A. Heiberger, and M. D. Kramer. 1989. Cloning and sequencing of the gene encoding the outer surface protein A (OspA) of an European Borrelia burgdorferi isolate. Nucleic Acids Res. 21:8864.

26. Bergstrom, S., V. G. Bundoc, and A. G. Barbour. 1989. Molecular analysis of linear plasmid-encoded major surface proteins, OspA and OspB, of the Lyme disease spirochaete Borrelia burgdorferi. Mol. Microbiol. 3:479-486.

27. Arnett, F. C., S. M. Edworthy, D. A. Bloch, D. J. McShane, J. F. Fries, N. S. Cooper, L. A. Healey, S. R. Kaplan, M. H. Liang, H. S. Luthra, et al. 1988 The American Rheumatism Association 1987 revised criteria for the classification of rheumatoid arthritis. Arthritis Rheum. 31:315-324.

28. Dressler, F., N. H. Yoshinari, and A. C. Steere. 1990. The T cell proliferative assay as a diagnostic test for seronegative Lyme disease. Arthritis Rheum. 33:S 36. (Abstr.)

29. Bressan, G. M., and K. K. Stanley. 1987. pUEX, a bacterial expression vector related to pEX with universal host specifity. Nucleic Acids Res. 15:10056.

30. Preac-Mursic, V., B. Wilske, and G. Schierz. 1986. European Borrelia burgdorferi isolated from humans and ticks. Culture conditions and antibiotic susceptibility. Zentralbl. Bakteriol. Mikrobiol. Hyg. Ser. A. 263:112-118.

31. Wilske, B., V. Preac-Mursic, G. Schierz, R. Kühberg, A. G. Barbour, and M. Kramer. 1988. Antigenetic variability of Borrelia burgdorferi. Ann. NY Acad. Sci. 539:126-143.

32. Laemmli, U. K. 1970. Cleavage of structural proteins during the assembly of the head of bacteriophage T4. Nature (Lond.). 227:680-685.

33. Steere, A. C., E. Dwyer, and R. Winchester. 1990. Association of chronic Lyme arthritis with HLA-DR4 and HLA-DR2 alleles. N. Engl. J. Med. 323:219223.

34. Cooke, W., B. J. Luft, T. F. McNamara, M. Golightly, P. D. Gorevic, and R. J. Dattwyler. 1990. Antibodies to Borrelia burgdorferi recognize epitopes on Treponema denticola. Arthritis Rheum. 33:S84. (Abstr.) 